\title{
Using Different Network Technologies and Wireless Sensor Networks to Design and Implement a Fully Smart Home System
}

\author{
Bilal Naji Alhasnawi \\ Electric Eng. \\ University of Basrah \\ Basra/ Iraq
}

\author{
Basil H. Jasim, PhD \\ Electric Eng. \\ University of Basrah \\ Basra/ Iraq
}

\begin{abstract}
This paper presents a thorough explanation of diverse, smart homes systems and technologies from the viewpoint of control and safety. This work highlights numerous faults with regard to safety in current smart home systems. Various smart homes machineries are considered in this project, including Internet-based, Short Messaging Service-based, mobile Global System for Mobile communications-based, Bluetoothbased, and Email-based smart home systems. The proposed system is made up of two parts: the hardware and software. The hardware consists of a base station unit (BSU) and several terminal nodes (TNs). The BSU is comprised of the main unit, represented by a Raspberry $\mathrm{Pi} 3$, while the $\mathrm{TN}$ represented by a Wemos-D1 board, the required sensors and appliances. The software is made up of the programming of the Wi-Fi network and the system protocol. In this paper, an MQTT (Message Queue Telemetry Transportation) broker was built on the Raspberry Pi3 and Wemos-D1. The MQTT broker was utilized as a platform to provide the Internet of Things (IoT) services, which control and monitor smart home appliances. The benefit of the GSM, Internet, and Email is that the home device can be controlled from anywhere in the world.
\end{abstract}

\section{Keywords}

Raspberry Pi3; E-mail; IoT; Android application to Bluetooth; GSM; Wemose-d1

\section{INTRODUCTION}

Wireless sensor networks (WSNs) are comprised of terminal nodes that monitor the statistics of things in their coverage zones. The information and sense of the nodes are transmitted indirectly or directly to the Base Station (BS) or sink in a wireless network [1]. The fault tolerance and precision of WSNs are increased via dispersed processing, and therefore, they have broad request prospects in industries, the army, daily life and gardening [2]. The IoT covers several networks of physical objects with actuation and sensing embedded units. Under the hood, IoT uses multiple network protocols to communicate between devices. For example, different components in the physical location share information or actuate based on information received through network communications. One of the fields that have benefitted from the IoT is the smart home. The smart home uses different types of network protocols such as Wi-Fi, ZigBee and Bluetooth [3]. The constant growth in mobile technology and rapid developments in embedded systems have made it possible to integrate mobile technology into the design of smart home systems. The smart home enables several house devices to be monitored and controlled via a unique system, and it provides household customers with better suitability, greater safety, and greater energy efficiency. The integration of smart home systems into smart grids in the future will give clients the ability to control their home system and save energy efficiently. Recently, domiciliary energy management has become an active research topic [4][5]. The involvement of smart grids in building and home automation systems has led to the growth of different standards for interoperable products to control devices, lighting, security and energy management. The smart grid enables users to control the energy usage according to the demand and price. This work generally centres on the control and safety aspects of the smart home. The paper goes on to discuss numerous smart home systems, followed by the subject of their safety, as established by the method utilized, namely, a context description smart home system, crucial regulator-based smart home system, Internet-based smart home system, GSM or mobile-based smart home system, Bluetooth-based smart home system, Email-based smart home system, and a dispersed approach to smart home systems. Lastly, the employment of consumer interfaces with regard to safety is discussed, and the paper ends with the conclusions drawn from the project with regard to the control and safety of smart home systems.

\section{LITERATURE REVIEW}

In [6], the definitions for the home automation devices and interfaces were presented to explain the interoperability amongst ZigBee appliances via numerous electrical tools, intelligent energy producers, and meters. A smart, selfregulating sensor for a home automation system service station was established on ZigBee Communications and presented in[7]. In [8], the MQTT Publish/Subscribe Protocol and Django Web Framework were designed to give users the capability to integrate many open-source devices with opensource tools and to optimize mobile sites. A. Alheraish[9] proposed a system of smart homes using SMS (Short Message Service). The proposed system senses illegal interruptions in the house and permits valid customers to adjust the key for controlling the lights and the door to the house. The entry of a thief into the home is detected by monitoring the state of the house door, which comes complete with infrared sensors and an LED. The key to the door can be a combination of four digits, which can be set both by utilizing the SMS or the keypad from a recorded customer's phone number. A customer can regulate the lamps in the house remotely by utilizing an SMS message after their recorded phone number, thereby turning the lamps on in different rooms at chance intervals of time, which is a unique way of giving the impression that the house is occupied, even when it is not. M.S.H Khiyal et al. [10] proposed an SMS-based system for 
house safety called an SMS-established Wireless HACS (Household Appliance Control System). In this system, a house owner is able to control the house by utilizing an SMS from a pre-set recorded phone number. If the SMS message is not from a genuine phone number, the system will disregard the SMS. In case of an interruption, the device regulator subsystem and safety subsystem in the proposed system will notify the homeowner through an SMS message. U. Saeed et al [11] also proposed an SMS-based smart home system. The system has a request for a Java application on the telephone. Genuine customers can $\log$ in to the application using their password, username and can select the structure/room/floor/appliance that they want to control remotely, along with an appropriate action from the list of available customer actions. The Java application will create an appropriate SMS message and direct it to the GSM in the house. The GSM will receive the SMS message, decipher it, and license it to the house network to implement the identified act. The student's utilized facial recognition and a 5-digit key for safety. A.R Delgado et al.[12] utilized a General Packet Radio Service (GPRS) communication as a backup to an Internet-based smart home system. This enhanced the fault tolerance of the system. House owners are alerted by alarms on their telephones about unusual modifications to conditions detected by the sensors. The customer can then take action, either by utilizing a web interface or messaging. In any case, there will be two possible techniques to access the house, so if one fails; the customer can depend on the other. $\mathrm{N}$. Sriskanthan et al. illustrated the operation of a smart home system utilizing Bluetooth. They applied a host controller to a personal computer, which was linked to microcontrollerdependent device controllers and sensors. The researchers even built a new protocol on top of the Bluetooth stack software, called HAP (Home Automation Protocol), to make the communication between appliances possible. The appliance controller is linked to the electronic appliances through the I $2 \mathrm{C}$ Bus. The system allows more than one appliance controller to be related to the host regulator [13]. $\mathrm{H}$. Kanma et al. [14] also proposed a smart home system utilizing Bluetooth that can be accessed remotely through GPRS. The investigators utilized a cell phone armed with a GSM modem that provides Internet and Bluetooth connectivity as a host controller. The home appliances are fitted with Bluetooth communication adapters so that they can communicate with the host controller telephone by Bluetooth. The paper discussed the remote updating and controlling of house appliances, along with the detection and fault diagnostics. The project also discussed providing an electronic customer manual on the telephone via the Internet and Bluetooth.

\section{SYSTEM OVERVIEW}

This project was motivated by the use of modern machinery to help control smart homes or, in other words, various methods of home control automation. The proposed smart home system is a flexible system that can control and create a link between nearly all the loading devices in a home. All the devices can be controlled from both the outdoors and indoors from any place. This smart home system can be called an automated home system. If a person forgets to switch on the lights or another device when going out, it allows him to turn off the device with an SMS from his mobile or Internet web page. By using an Internet web page or mobile phone SMS, a user will be able to obtain the status of his house and will also be able to control the power appliances in his house. The smart home system is implemented using an Internet module for the web page, GSM-SMS, Email and a Bluetooth. First, the internet module was set up using a dashboard web page, followed by the GSM-SMS, Email and Bluetooth. The main advantages of this system are:

1- The user can control the devices through the internet web page from any place.

2- Control is by GSM (SMS), where the appliance is capable of recognizing the customer.

3- Control is by Email, where the appliance is capable of recognizing the customer.

4- Control is through an Android application for mobile phones.

The first, second and third mechanisms are suitable for controlling house devices from anywhere in the world, while the fourth mechanism is only applicable inside the house. In the Internet web page, GSM, Email, and Bluetooth, the house can be designed in zones. Each zone must contain at least one node (including the sensing of the required parameters and the controlling appliances). These nodes must communicate with the BSU, while the growth of all the advantages of this smart system is being completed. The proposed design used the MQTT Publish/Subscribe Protocol, node-red and Python to give the client the capability to integrate many open source tools with open-source devices and mobile site optimizations. The MQTT Publish/Subscribe Protocol is used between machines that have the capability of connecting to a network (between Raspberry Pi3 B and ESP8266 for Wemos-D1, and between Raspberry Pi3 and node-red dashboard). The Raspberry Pi3 Service Interface Programming messages can be replaced utilizing MQTT. The central benefit is that clients can join their requirements to numerous nodes: as a replacement for opening a socket for each microcontroller, the client can simply publish and subscribe messages to a broker. Similarly, the MQTT bridges forward serial messages from the board to publish actions for appropriate topics and to route subscribed messages to the serial channel [15].

\section{SYSTEM HARDWARE DESIGN}

The system hardware consists of a single Base Station Unit and numerous Terminal node. The details are as follows:

\subsection{The Base Station Unit}

The base station unit (BSU) plays an important role in the proposed system. The BSU represents the network or the system coordinator. The hardware of the base station consists of a Raspberry pi3 board. Figure 1a shows a block diagram of the system base station, Figure 1b shows the internal structure of a prototype BSU that used for implementing the system.
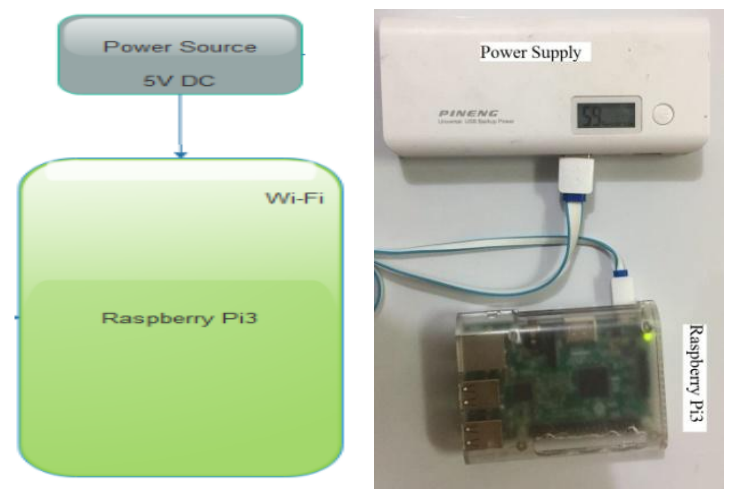

Figure 1: (a) schematic diagram of the BSU, (b) system BSU internal structure 


\subsubsection{The Raspberry pi3 Board}

The RaspberryPi is a sequence of credit card sized single board computer. All RaspberryPi model advantages a Broadcom system on a chip (SoC), which includes an ARMcompatible CPU and an on-chip GPU. Since Raspberry Pi is actually not a microcontroller, but a small-size and fully functional computer, it offers very good computational powers. With CPU speed ranges from $\left(700 \mathrm{MHz}_{-} 1.2 \mathrm{GHz}\right)$, and on board RAM of $1 \mathrm{~GB}$, the Raspberry Pi is powerful enough for every complex control operation that would be required at nodes. Though it does not have on- board Flash Memory, an external SD card should be used with the capability from 8 - $16 \mathrm{~GB}$ allows to store multiple programs and even images from security camera module if needed. The Raspberry Pi could also load and run Linux Operating System from micro SD card. The Linux OS allows the Raspberry Pi to do multitasking, and provide a lot of flexibility and easiness when dealing with the real-time requirement of the system. Raspberry Pi also offers 40 general purpose GPIO pins. Moreover, the new version of Raspberry Pi 3 Model B released in February 2016 provides built-in Wi-Fi, which reduces a lot of works and costs buying and installing a separate Wi-Fi module to microcontrollers. [16][17].

\subsection{The Terminal Units}

The Terminal Units (TUs) are the microcontroller system that responsible for the measurements of temperature humidity, gas, flame, water level and light intensity depending on the sensors content in the node. The LM35 sensor is used for sensing the surrounding relative temperature in the bedroom field; The DHT-22 sensor is used for sensing the relative humidity and the temperature in the living room field. The LDR sensor is used for sensing the surrounding relative light intensity in the garden field; The motion sensor is used for sensing motion in the office field and the ultrasonic sensor used for detecting the water level in the tank.

The controller of the nodes is a Wemose- $\mathrm{d} 1$ board which is responsible for gathering and processing sensors data and sending the obtained information to BSU. Figure 2 shows the internal structure of a prototype TUs that used for implementing the system.

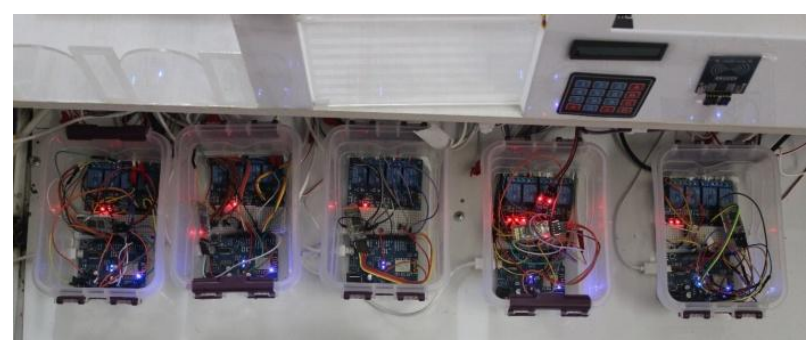

Figure 2: The internal structure of the system TUs

The TU (Wemos-d1 board) is configured by the Arduino (IDE) to be the coordinator of end devices. By supporting the Arduino (IDE) which supported by the $\mathrm{C}$ or $\mathrm{C}^{++}$, the ESP8266 microcontroller that content in the Wemose-d1 board is programmed for supporting the proposed protocol of the system.

\section{A) Wemose-d1 board}

Wemose-d1 board is high-performance, open-source microcontroller boards based on flexible, easy to employ software and hardware. The language is C. Wemose-D1 board is built with an ESP8266 microcontroller. It consists of 14 input/output digital pins, 1 analog input, $1 \mathrm{~K}$ Bytes EEPROM, $2 \mathrm{~K}$ bytes SRAM, $32 \mathrm{~K}$ bytes ISP flash memory, $80 \mathrm{MHz}$ crystal oscillator, serial communication UART represented in pin 0 (RX) and pin 1 (TX), SPI serial port defined in GPIO10 which represents select slave (SS) GPIO, GPIO11 which is the master out slave in (MOSI) GPIO, GPIO12 specified to the master in slave out (MISO) GPIO and GPIO13 is the serial clock (SCK) GPIO. The Wemos-d1 board can be powered through the USB connector, AC to DC adapter or using the battery [18].

Also, the Wemose-d1 is a great platform for any smart home system. Can create an MQTT (Message Queue Telemetry Transportation) communication, control outputs, read inputs and interrupts.

\section{1) The First TU (the Bedroom)}

The first $\mathrm{TU}$ is responsible for the measurements of temperature and control (turn on/off) of light, Air condition and control (open/close) door and window. The LM35 sensor is used for sensing the temperature. Figure 3 shows the schematic diagram of the first TU.

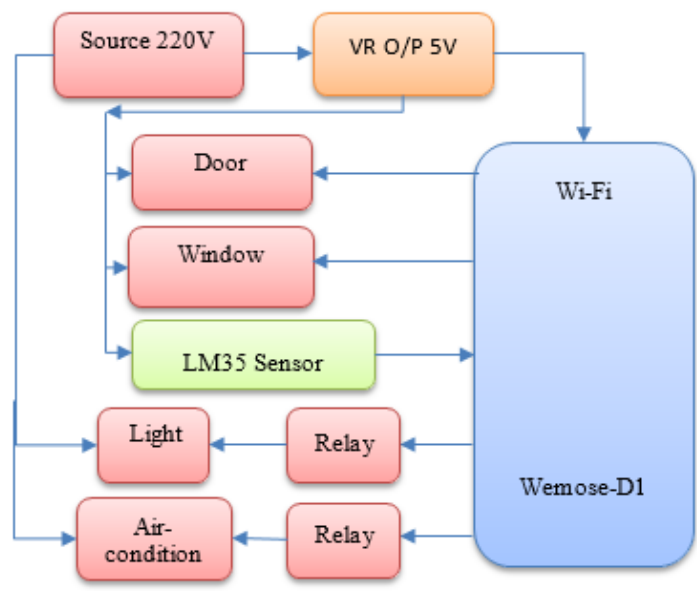

Figure 3: The system first node schematic diagram

2) The Second TU (the hall, garden and water tank).

The second TU is responsible for the measurements of the Water level of the tank, the intensity of the garden light. Control (turn on/off) of garden light, water pump, hall light. Control (open/close) hall door. The ultrasonic sensor is used for sensing the Water level of the tank. The LDR sensor is used for sensing the intensity of the garden light. Figure 4 shows the schematic diagram of a system node.

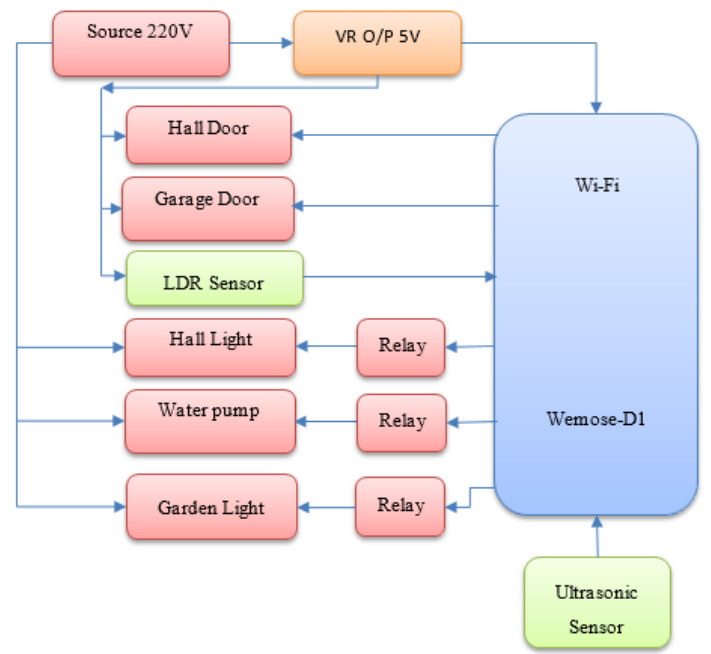

Figure 4: The system second node schematic diagram 


\section{A) water level Sensor Interfacing}

To measure the water level in tank Ultrasonic sensor is used to measure the distance from the top of the water level. an ultrasonic transmitter is mounted on the top of the tank and transmits an ultrasonic pulse down into the tank. This pulse, which travels at the speed of sound, will be reflected back to the transmitter from the liquid surface. The time delay between transmitted and received signals enables the program to calculate the distance to the surface.

\subsubsection{The Third TU (the living room)}

The third TU is responsible for the measurements of temperature, humidity, control (turn on/off) of light, fan, control (open/close) door and window. The DHT11 sensor is used for sensing the temperature and humidity. Figure 5 shows the schematic diagram of a system node.

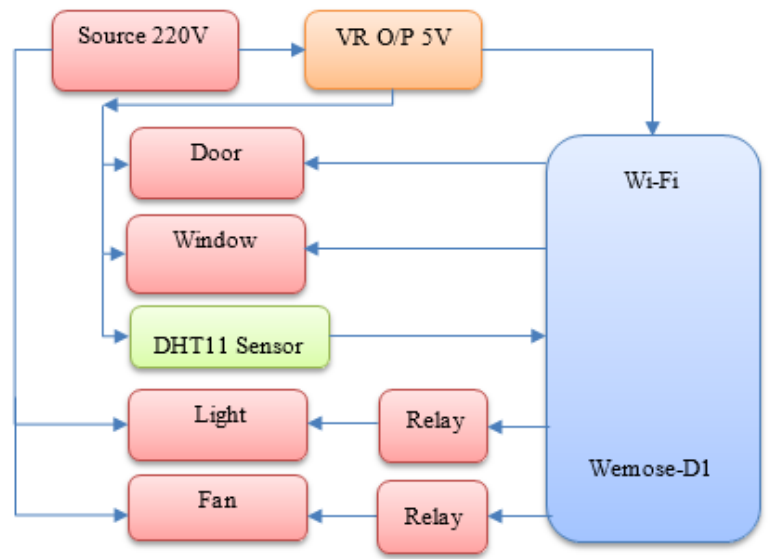

Figure 5: The system third node schematic diagram

\section{4) The forth $T U$ (the kitchen)}

The fourth TU responsible for the measurements of gas and flame, Control (turn on/off) of light, refrigerator, Control (open/close) kitchen doors and windows. Figure 6, shows the schematic diagram of a system node.

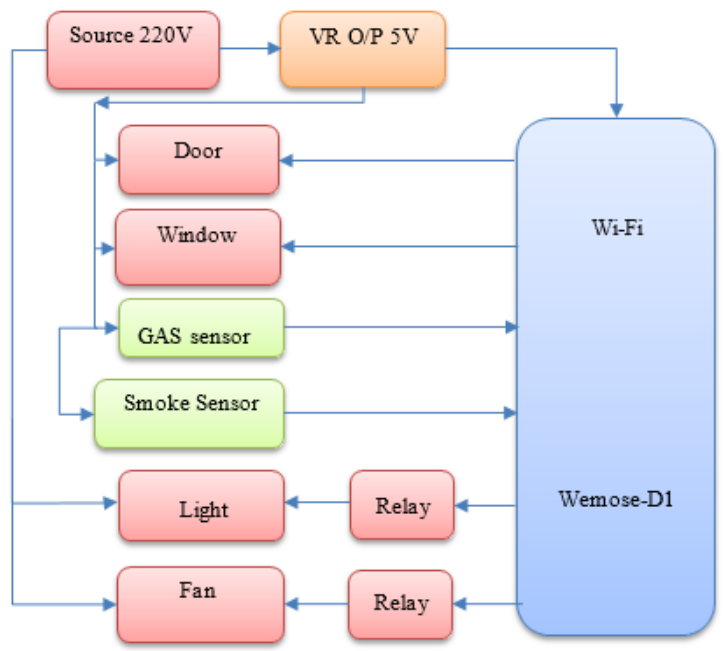

Figure 6: the system forth node schematic diagram

\section{5) The fifth $\mathrm{TU}$ (the office)}

The fifth TU responsible for the measurements of office motion, Control (turn on/off) of light, computer, Control (open/close) office doors. Figure 7 shows the schematic diagram of a system node.

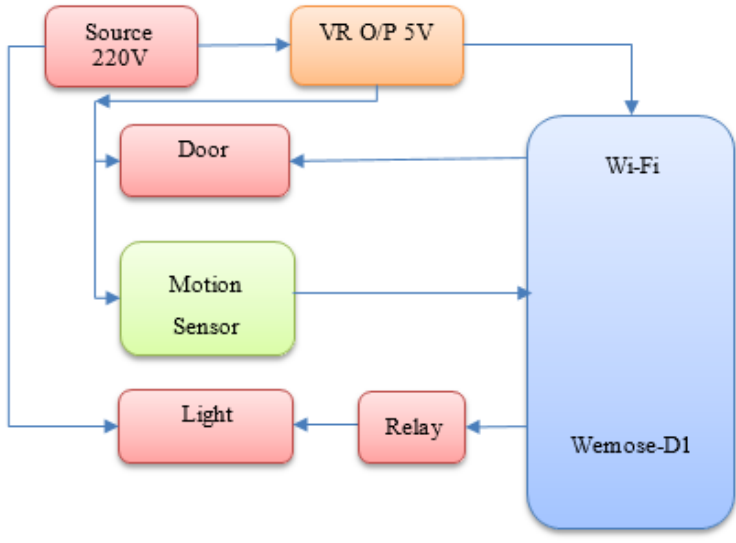

Figure 7: The system fifth node schematic diagram

\section{HARDWARE IMPLEMENTATION}

The prototype design aims to assemble all of the components of the smart house, including servo motors, Raspberry Pi3, Wemose-d1 board, lights, and sensors. The prototype design consists of the bedroom, living room, kitchen, office, hall, garden, water tank, and car garage.

In this prototype, nine servo motors were used, which are responsible for closing and opening the doors and windows. Servo motor 1 was installed on the door of the bedroom to open and close the door, whereas servo motor 2 was installed on the window of the bedroom to open and close the window, as shown in Figure 8 Below.

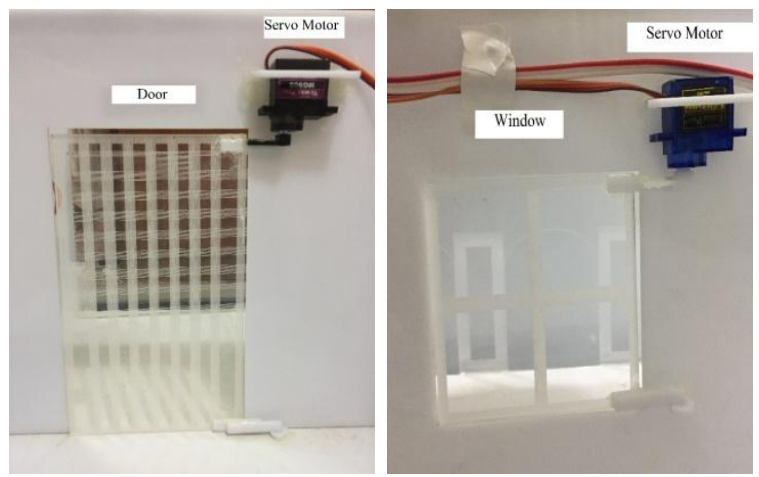

Figure 8: The process of installing the servo motor on door and window

The whole system was assembled in the smart house prototype, which was designed to accommodate all of the hardware components. The final design of the smart house system is illustrated in Figure 9.

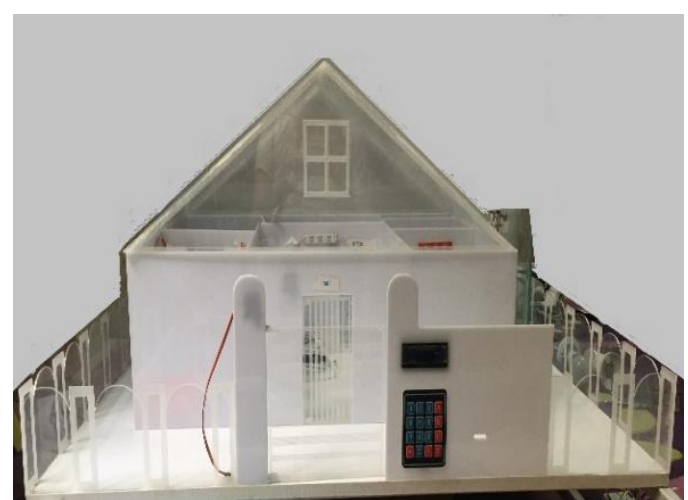

Figure 9: (a) and (b) show the final design of the smart house prototype 


\section{PROPOSED SYSTEMS METHODOLOGIES 6.1 Smart Home through Internet Web page}

The internet of things (IoT) has meaningful efficient to transform business. IoT is an inspiring technology which allows different things and devices to be controlled with the internet. At its heart, IoT is an extensive-ranging ecosystem of everyday anatomical objects connected to the Internet, capable of identifying themselves and informing data to the objects on the internet network. In this work, it is implemented using the Raspberry pi3 hardware as a web server which will connect the Wi-Fi module through which can connect to the hardware's and receive status updates from them and then send control information to the microcontroller of TU.

In this part, smart home different activities are managed by internet web page. All appliances can be controlled from indoor and outdoor from any place. Also, all home parameters and status (sensed physicals values such as temperature and the status (on/off) of appliances) can be gotten from this web page.

\subsubsection{Methodology}

In this work, home appliances are controlled by a command from user's web page. The commands are used to control the appliances.

The computer, tablet, smartphone, send information to the BSU module by Wi-Fi network. Then, BSU sends a command to nodes. This flow also works the other way around: The microcontroller of TU sends information to the BSU. BSU sent information to the smartphone, computer, and tablet through a web page by Wi-Fi.

In this work, a point-to-multipoint topology is used. The nodred software has been used for raspberry pi3 module configuration for building the wireless Wi-Fi network. In this network, all nodes are managed by BSU. Figure 11, shows part of the BSU configuration by using the Node-Red software.

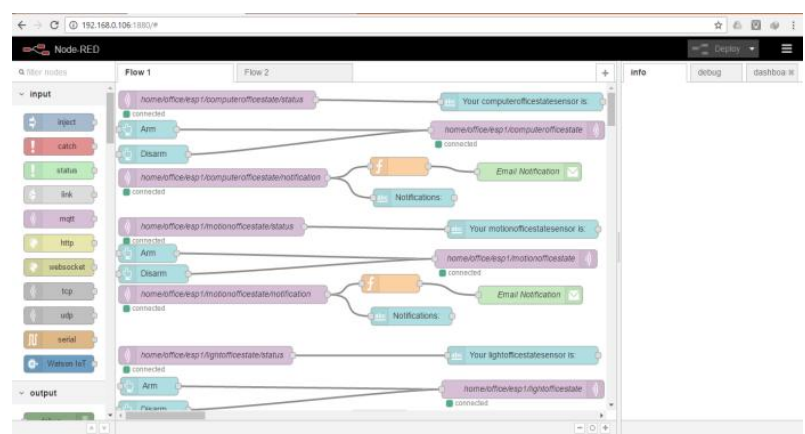

Figure 11: The BSU configuration by using the Node-Red software
To provide greater detail, it was assumed that each microcontroller board of TU was identified via a single name, and the following scheme was employed for the topics:

1) Messages, from a definite board, are published by the board into MQTT broker using the topic, esp/node name/out. The microcontroller is identified by node name.

2) The client Messages are published to the particular board by the MQTT broker with the topic, esp/node name/in.

MQTT bridges of a node most subscribe to the topic, esp/node name/in. forward messages received on this topic through the serial connection. The same bridge most publish serial messages from the node into the topic, esp/node name/out. Conversely, specific node client would subscribe to the topic, esp/node name/out, to accept messages from the node, and publish messages to esp/node name/in to send messages to the node. Client's applications and MQTT bridges are available in the node-red blocks. Figure 12: show overview of the internet system

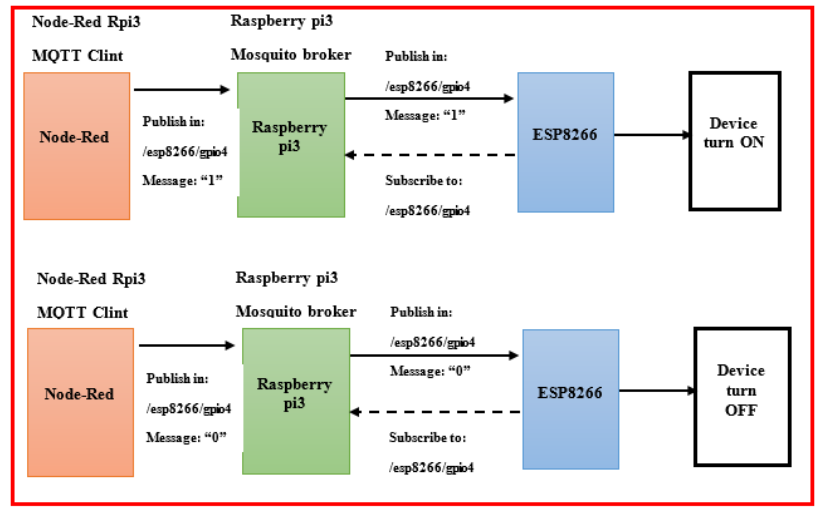

Figure: 12 Show overview of the internet system

\subsubsection{The Web Page System Protocol}

After executing the program, the BSU Receives command from a Web page, BSU sends the command with an address to a zone node. The BSU receives the sensed data from all zone and sends these data to the web page. Each TUs receive the addresses from the BSU, if the TU address matches the received address, then it will interact with the received message and do the commands contained in it if any. Figure 13a shows a flowchart of the BSU proposed web page system. And the TU flowchart is explained in Figure 13b. 


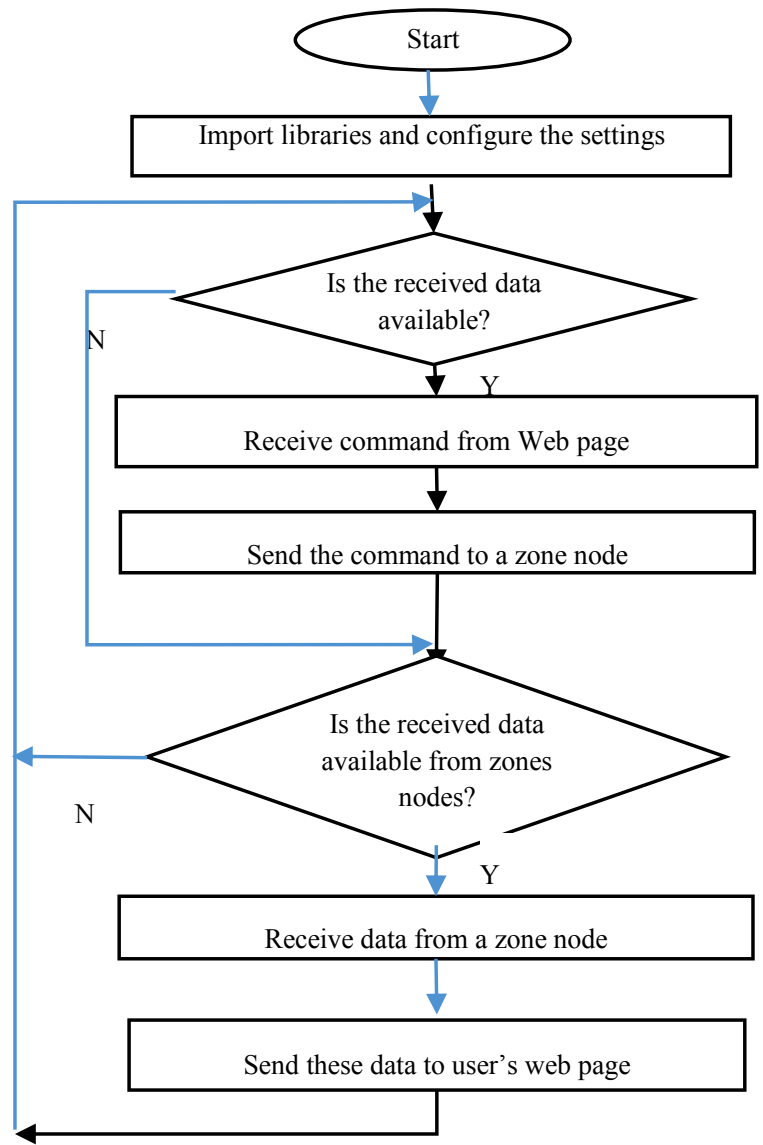

(a)

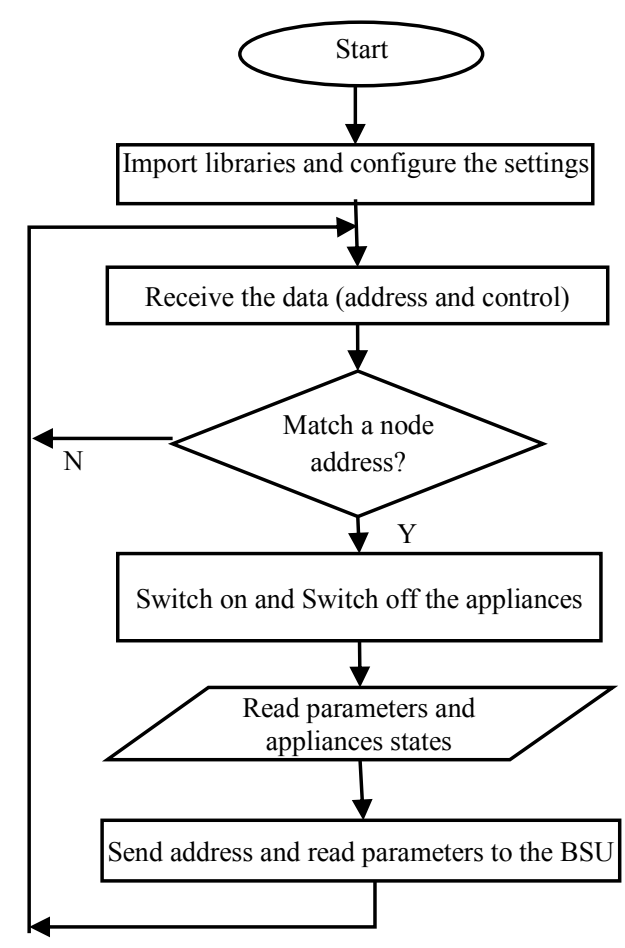

(b)

Figure 13: show BSU and TU for Web page technology (a) The BSU flowchart. (b) SU flowchart.

\subsubsection{Internet Access from anywhere}

For local access of Web page the IP used is the local Raspberry Pi IP with the port 1880 which had been dedicated for Node-Red for a web page. Then the local IP is http://192.168.0.106:1880/ui. to broadcast the web page over the internet, Ngrok application is used. Ngrok helps in converting the local IP address of raspberry pi3 to a global IP address that can be accessed from anywhere in the world over the internet. Access the web page from the internet, the address of Ngrok server is HTTP:// 55cba350.ngrok.io.

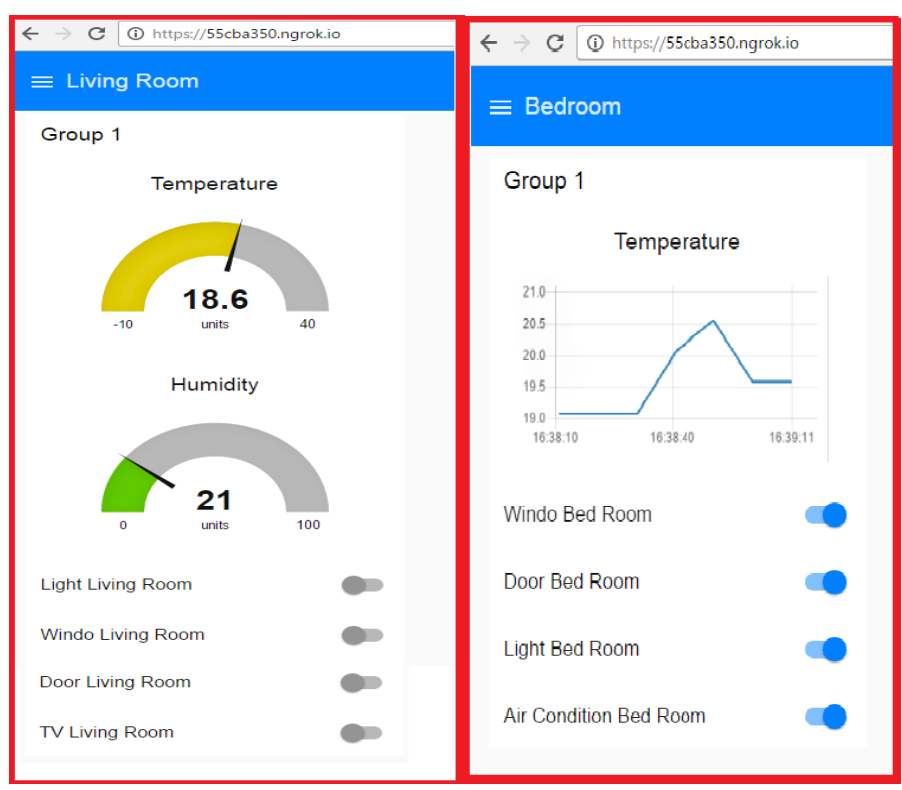

(a)

(b)

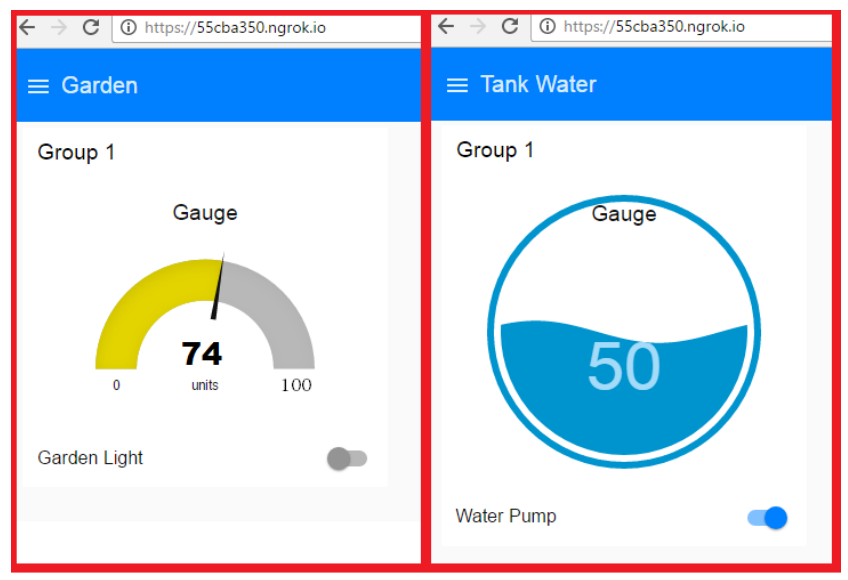

(c)

(d)

Figure 14: internet web page: (a) Contents of the living room. (b) Contents of the bedroom. (c) Contents of the garden. (d) Contents of the water tank.

\subsection{Smart home activities using GSM}

In tahis part, a system capable of controlling home appliances and sending notices using SMS has been developed.

The aim of this part is to develop a smart home application using Raspberry pi3, Wemose-d1 and GSM. Programming has been developed in $\mathrm{C}++$ in Wemose-d1 and Python environment for Raspberry pi3 operation. The MQTT technologic used to connect between BSU (Raspberry pi3) and TU. 


\subsubsection{Methodology}

In this work, home appliances are controlled by SMS from user's mobile.

The remote client sends SMS commands to the receiver Such as ON/OFF commands to appliances. GSM receiver gets messages sent from client cell phone. Then, the GSM module sends the commands via serial communication to the BSU. Then the BSU send the command to TU. The TU checks for status and applies operation on electrical appliances. This flow also works the other way around: the microcontroller sends information to the BSU and wich sends to GSM. Then GSM sent this information to the owner phone.

In this work, a smart system is designed and implemented for home management. There are some features that are available in GSM communication which make it suitable for the proposed system like the small size, low cost, an emergency alarm generated, very short response time and the main feature is the wide area coverage. So the user can interact with the system even from anywhere. Figure 16 shows an overview of the GSM system.

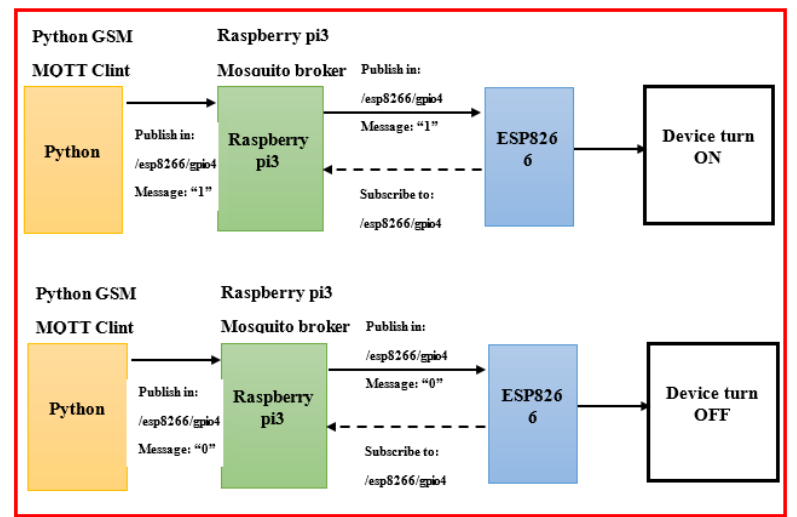

Figure 16 Show overview of the GSM system

\subsubsection{GSM SIM 900A Interfacing with BSU}

The Raspberry Pi3 (BSU) and GSM were connected via an RS232 serial communication protocol. AT commands have been used to program the GSM module for sending and receiving SMS. The interfacing of the GSM to the Raspberry pi3 board shown is in Figure 17a. The practical hardware of GSM with Raspberry Pi shown is in Figure 17b.

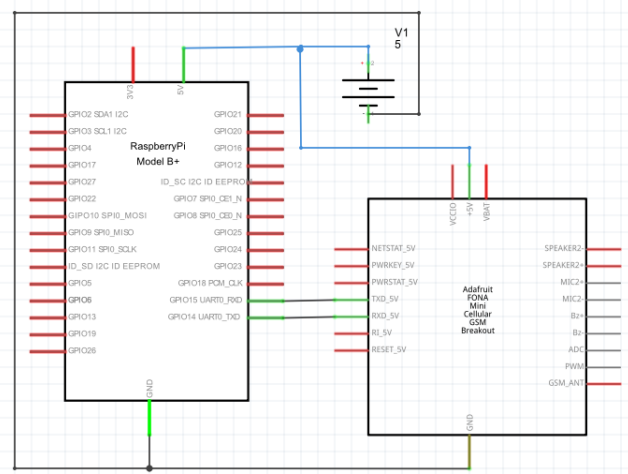

(a)

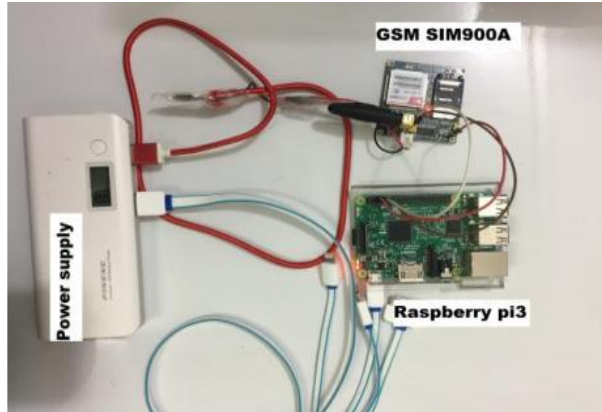

(b)

Figure 17: (a) Interfacing GSM with Raspberry Pi board, (b) Internal construction

\subsubsection{Software Description}

Python has been used to program GSM model using AT command. Figure 18, shows part of the base station configuration by using Python software

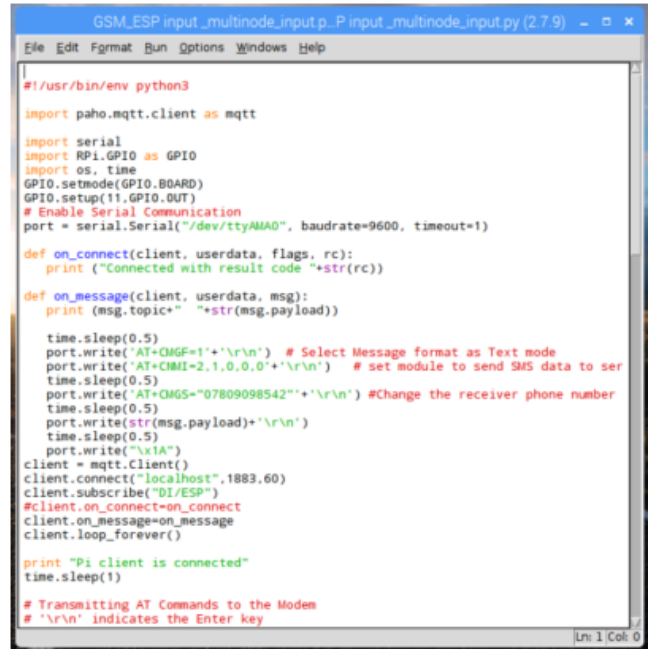

Figure 18: The base station configuration by using Python software

The general protocol used for sending and receiving SMS command can be described as following. The GSM receives a command from users mobile by SMS, the BSU receives a command from GSM, BSU Send the command with an address to a zone node. Each SU receives the address from the $\mathrm{BSU}$, if the SU address matches the BSU, it can control devices or measured parameter, then sends this parameter with its address to the BSU. The BSU Send these data to user's mobile. Figure 19a shows BSU flowchart of the proposed GSM system. And the SU flowchart is explained in Figure 19b. 


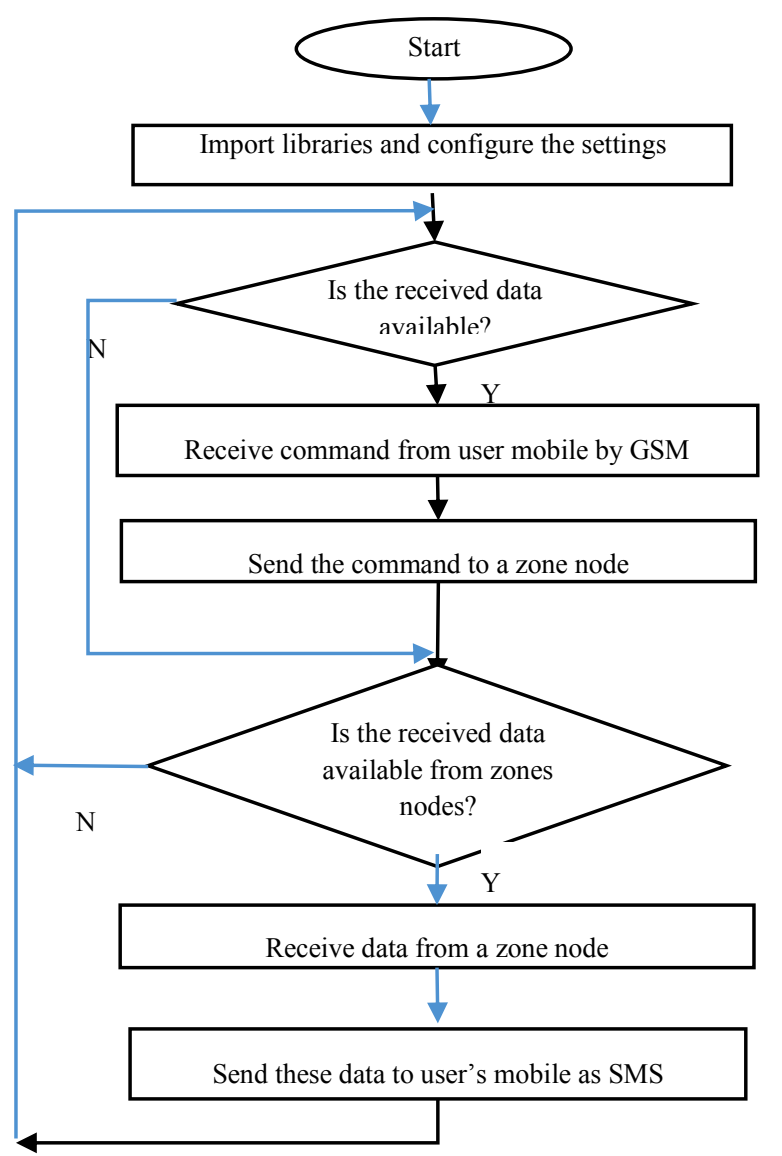

(a)

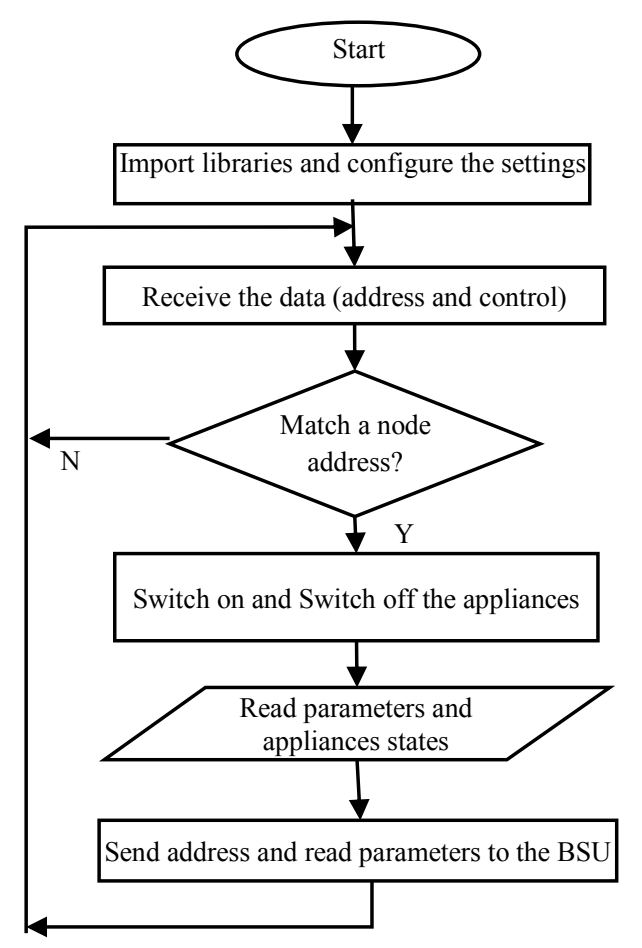

(b)

Figure 19: show BSU and TU for Web page technology (a) The BSU flowchart. (b) SU flowchart.

\subsubsection{The GSM System Testing in Prototype home}

The proposed GSM system was applied on prototype house.
The SMS command which is used by the owner to send control order is designed to be brief where it is split into three parts: zone, device and command (on/off). Zone part of the owner determine the zone numbers; the device part determines a device number in this zone and commands $(\mathrm{ON} / \mathrm{OFF})$ is represented by $(\mathrm{y} / \mathrm{n})$ respectively. As an example, If the user sends a message on GSM number such as "\#z1d1y" then lamp of the bedroom will be turned on for this message, and if a user sends a message such as "\#z1d1n", then lamp of the bedroom will be turned off.

Devices control (switch on and off) is performed by sending a code as SMS from a mobile code's formula is shown below:

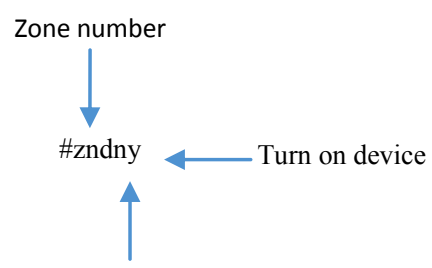

Device number

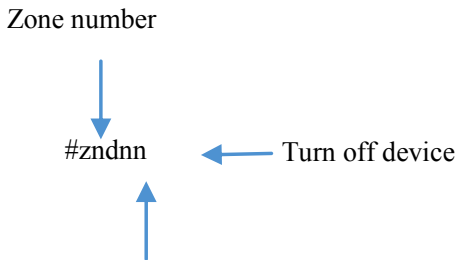

Device number

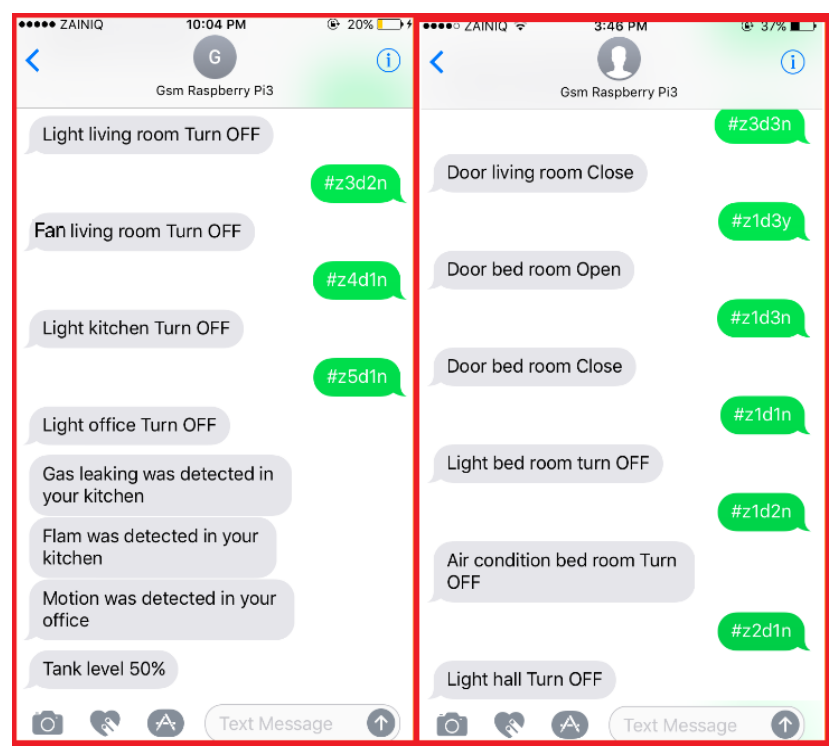

(a)

(b)
Figure 20: GSM Method: (a), (b), Turn ON, Turn OFF some home devices and monitor home devices.

\subsection{Smart home activities using Bluetooth}

The main objective of this part is to develop a home system using Android application for mobile with Bluetooth module. Now, people are expecting to control the home appliances through remote control. Bluetooth and Android OS (operating system) are used to develop a system which is used to control appliances and receive home parameters from sensors of TU. Modern houses are expected to have a centralized control system, instead of conventional switches which are fitted on walls. If the user wants to control the appliances, the user has to move near the wall and control the switches. So their difficulty to operate the appliances can be supported by the proposed home system which has a remote module to control the appliances. Remote control operation is achieved by a smartphone or Tablet which is supported by Android OS. In the smartphone, Android App with Bluetooth acts as a transmitter, which sends commands to the receiver Bluetooth module. This phone is used to control appliances using simple 
click commands. Commands are passing through the Bluetooth present in mobile.

In this work, Wemose-d1 controller board is used. The loads are interfaced to the Wemose-d1 controller using electromagnetic relays.

\subsubsection{Methodology}

MIT App Inventor, an Android App creator tool is used to design the GUI system in a smartphone. The GUI commands are used to control the appliances. In MIT app inverter, programming is based on using blocks to represent the required activates. This blocks should be programming to the required task. This App can be installed to Android phone, Tablet with Bluetooth module.

The smartphone sends information to the Bluetooth module via Bluetooth. Then, the Bluetooth module sends the information via serial communication to the microcontroller. This flow also works the other way around: the microcontroller sends information to the Bluetooth module that sends it to the smartphone via Bluetooth.

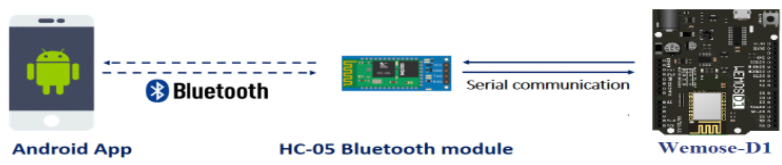

Figure 22: Explains how the information flows from the Android app to the microcontroller

\subsubsection{Bluetooth HC-05 Interfacing}

The Bluetooth HC-05 module is used in this part. This module uses an RS232 communication protocol to communicate with the host. Wemose-d1 board have one RS232 port which is used hear to interface HC-05 with the following siting: $9600 \mathrm{bit} / \mathrm{sec}, 8$ data bit, no parity, 1 stop bit. The interfacing of the Bluetooth HC-05 to the Wemose-d1 board is shown in Figure 23.

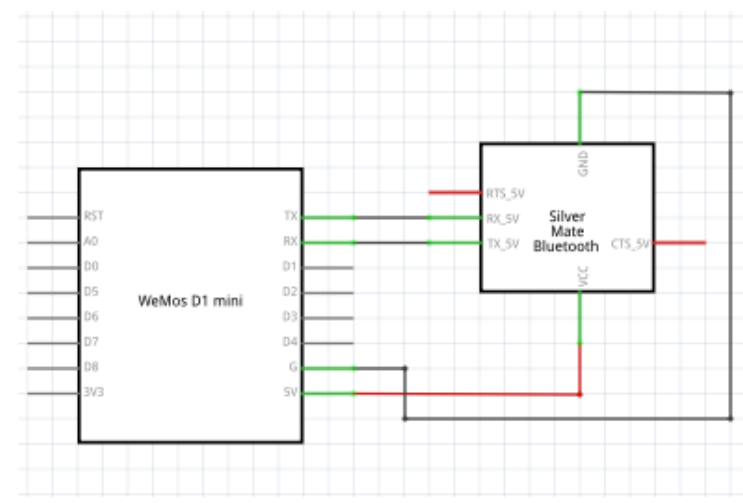

Figure 23: Interfacing GSM with Wemos-d1 board.

\subsubsection{The Bluetooth System Protocol}

The used protocol can be described as following, after powerup, if any command was sent from user mobile, HC-05 would Receive this command, and this command would be read by $\mathrm{TU}$, and the commands they are executed. The system is also continuously read sensors output and send them to the application on a smartphone via HC-05 Bluetooth module. Figure 24a shows a flowchart of the proposed Bluetooth system for first, second, third, fourth TUs. And the fifth TU flowchart is explained in Figure 24b.

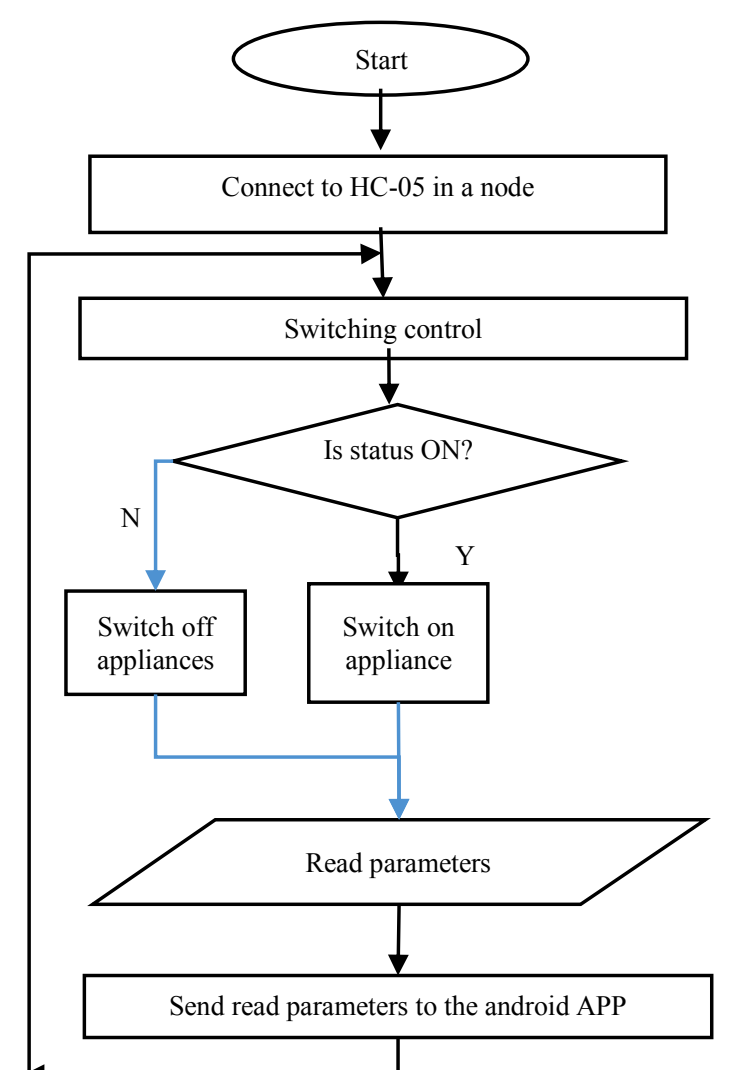

Figure 24: TU flowchart for Bluetooth technology

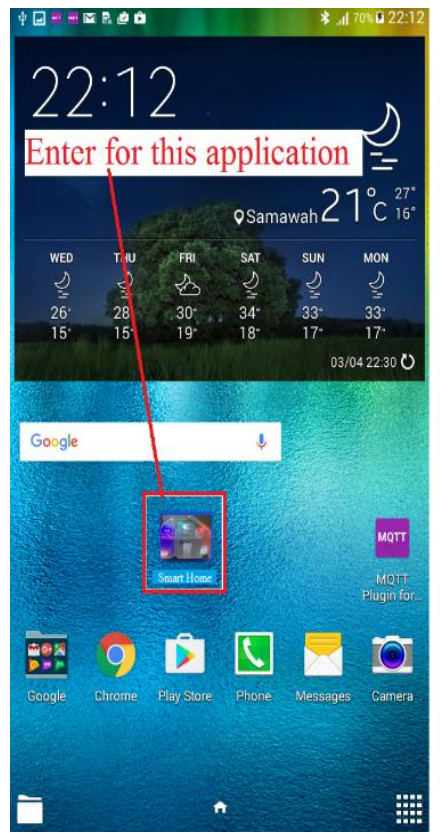

(a)

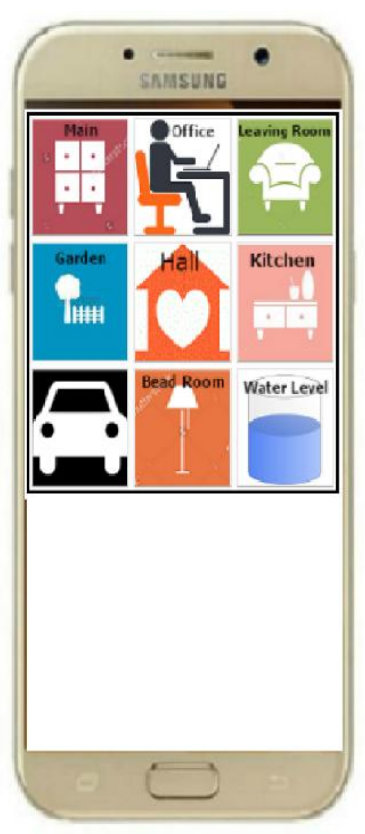

(b) 


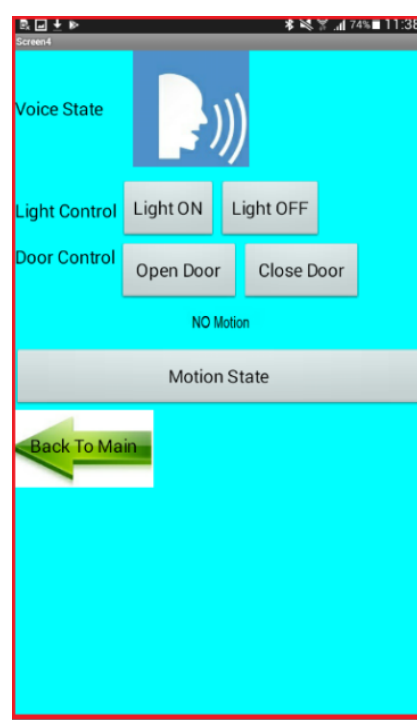

(c)

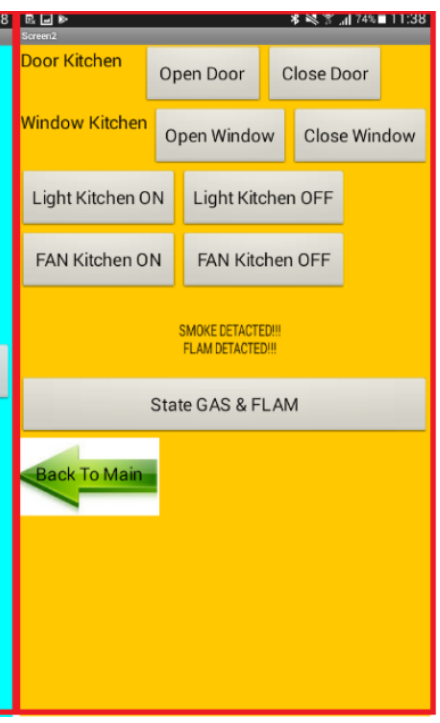

(d)
Figure 25: Bluetooth method: (a) Bluetooth application icon. (b) contains Bluetooth application. (c) Contents of the office icon. (d) Contents of the kitchen icon

\subsection{Devices control and receive notification through email}

In this part, the main objective is to develop a smart home system using Email. Figure 26 shown the structure of the proposed Email smart home system. Node-RED coded algorithm has been used to build an email server to receive and send Email. Every email received is checked by this application for being the owner email and if so then it is checked if any permitted command and if so, then the command is executed by sending the command accsotion TU also if any up normal condition happens then the application forms an notification email message which contains an information about this condition. This email is sent to the owner email.

Devices control (switch on and off) is performed by sending a code as email from an email code 's formula is shown below:-

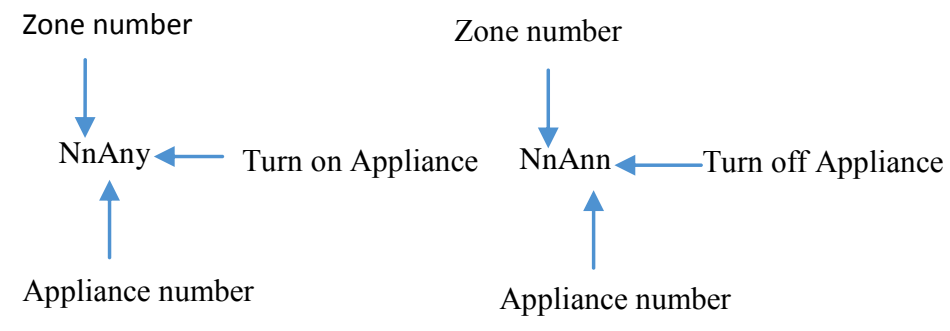

The flowcharts of email system for BSU and TU are as shown in Figure 27.

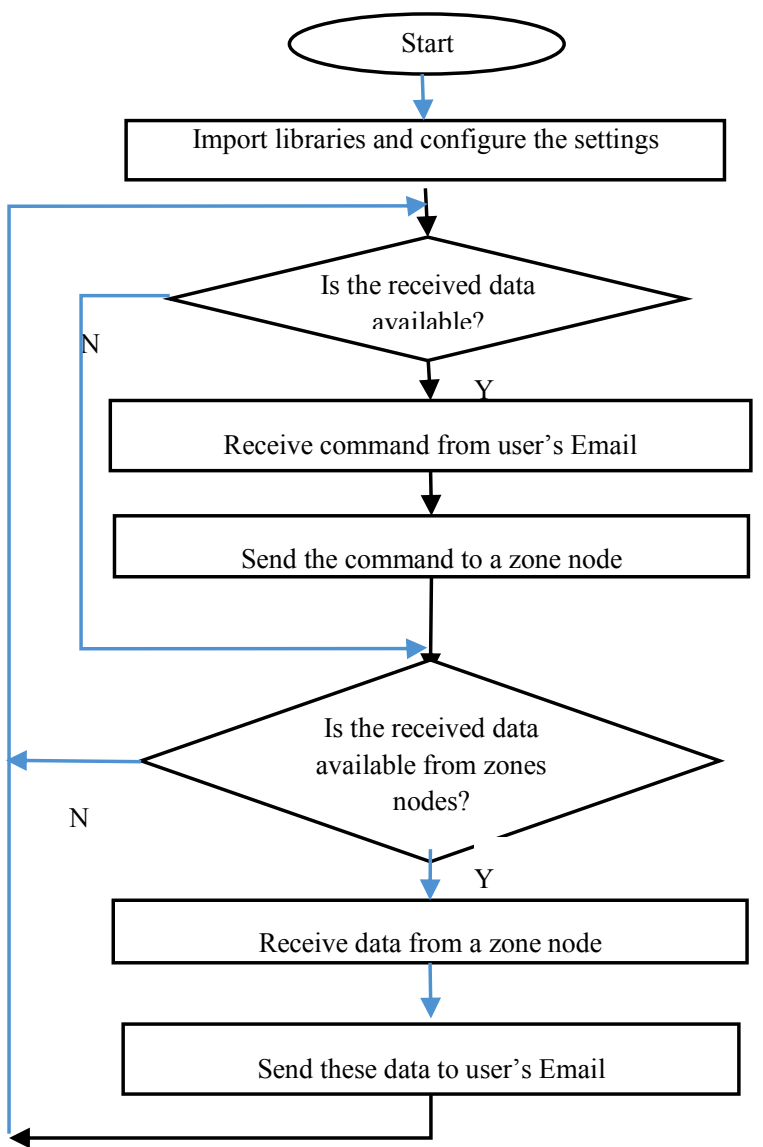

(a)

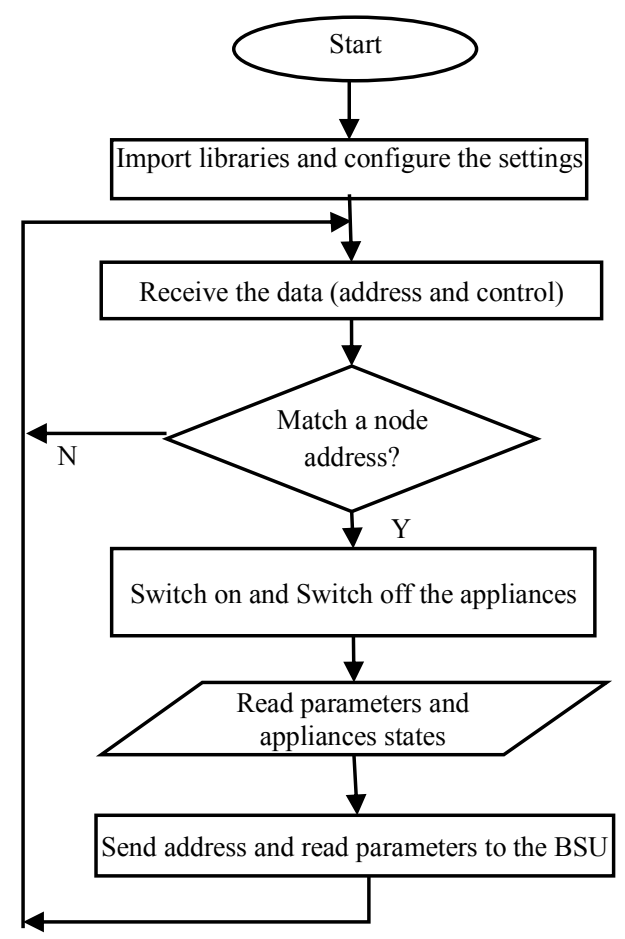

(b)

Figure 27: show BSU and TU for Web page technology (a) The BSU flowchart. (b) SU flowchart. 


\subsubsection{The Email System Testing in Prototype home} The proposed Email system was applied on prototype house. and in the actual field for measuring the required parameter. Control in lights, doors, windows and another device.

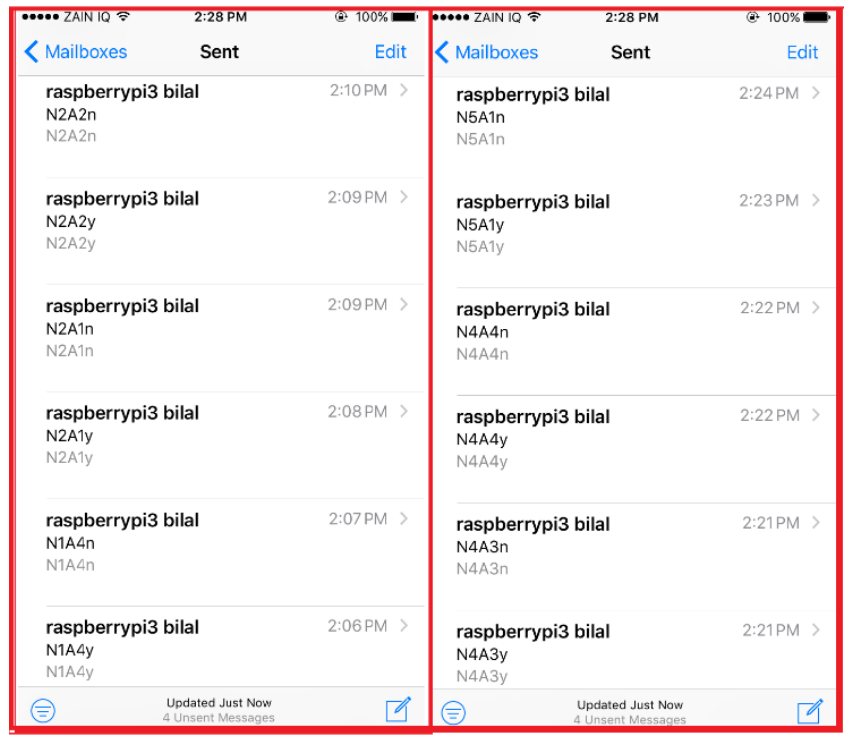

(a)

(b)

Figure 28 Show Email Method: (a), (b) Turn ON and Turn OFF some home devices

\section{DISCUSSION}

In this section comparison of all above-discussed home automation systems has been done and their all common features, advantages and disadvantages are highlighted. All above-discussed system has a main module that is connected to home appliances. Different types of communication techniques are used to transmit commands from the user interface to the main controller board. Comparison of cost, speed and real-time application of above-discussed systems is shown in Table 1.

Table 1. Comparison of different Smart Home System

\begin{tabular}{|l|l|l|l|}
\hline System & Cost & Speed & Real Time \\
\hline Internet & High & High & Yes \\
\hline GSM & High & Slow & NO \\
\hline Bluetooth & Low & High & Yes \\
\hline Email & Low & Slow & NO \\
\hline
\end{tabular}

\section{CONCLUSIONS}

This paper describes the design and implementation of a smart home system using numerous modern communication technologies. The system also offers a safety system. We proposed a wireless control and monitor system with wireless communication using sensors, ESP8266 Wi-Fi module on the Wemose-d1 and Mosquitto-MQTT broker on the Raspberry Pi3. The result showed that it could measure, transmit and receive the sensors rate data of the patients in real-time. The low bandwidth and low power consumption of MQTT protocol make this system practical for patients and low cost that quite suitable for rural or small house.

\section{REFERENCES}

[1] R. V Kulkarni, S. Member, A. Förster, and G. K. Venayagamoorthy, "Computational Intelligence in Wireless Sensor Networks: A Survey," Commun. Surv. Tutorials, IEEE, vol. 13, no. 1, pp. 68-96, 2011.

[2] J. Yick, B. Mukherjee, and D. Ghosal, "Wireless sensor network survey," Elsiver, Comput. Electr. Eng., vol. 52, no. 12, pp. 2292-2330, 2008.

[3] H. K. Ra, S. Jeong, H. J. Yoon, and S. H. Son, "SHAF: Framework for Smart Home Sensing and Actuation," in Proceedings - 2016 IEEE 22nd International Conference on Embedded and Real-Time Computing Systems and Applications, RTCSA 2016, 2016, vol. 10, no. 7, p. 258.

[4] A. H. Mohsenian-Rad and A. Leon-Garcia, "Optimal residential load control with price prediction in real-time electricity pricing environments," IEEE Trans. Smart Grid, vol. 1, no. 2, pp. 120-133, 2010.

[5] A. Molderink, V. Bakker, M. G. C. Bosman, J. L. Hurink, and G. J. M. Smit, "Management and control of domestic smart grid technology," IEEE Trans. Smart Grid, vol. 1, no. 2, pp. 109-119, 2010.

[6] D.-M. Han and J.-H. Lim, "Smart home energy management system using IEEE 802.15.4 and ZigBee," IEEE Trans. Consum. Electron., vol. 56, no. 3, pp. 1403 1410,2010

[7] J. Byun, B. Jeon, J. Noh, Y. Kim, and S. Park, "An intelligent self-adjusting sensor for smart home services based on ZigBee communications," IEEE Trans. Consum. Electron., vol. 58, no. 3, pp. 794-802, 2012.

[8] A. Alheraish and S. A. Dept. of Electr. Eng., King Saud Univ., Riyadh, "Design and implementation of home automation system," Ieee, pp. 1087-1092, 2016.

[9] A Alheraish, "Design and implementation of home automation system," Consum. Electron. IEEE Trans., vol. 50, no. 4, pp. 1087-1092, 2004.

[10] M. Sikandar, H. Khiyal, A. Khan, and E. Shehzadi, "SMS Based Wireless Home Appliance Control System (HACS) for Automating Appliances and Security Preliminaries Home Appliance Control System (HACS)," Issue s Sci. Inf. Technol., vol. 6, pp. 887-894, 2009.

[11] U. Saeed, S. Syed, S. Z. Qazi, N. Khan, A. Khan, and M. Babar, "Multi-advantage and security based home automation system," Proc. - UKSim 4th Eur. Model. Symp. Comput. Model. Simulation, EMS2010,IEEE, pp. $7-11,2010$.

[12] A R. Delgado, R. Picking, and V. Grout, "Remotecontrolled home automation systems with different network technologies," 6th Int. Netw. Conf. (INC 2006), pp. $357-366,2006$

[13] N. Sriskanthan, F. Tan, and A. Karande, "Bluetooth based home automation system," Microprocess. Microsystems, Elsevier, vol. 26, no. 6, pp. 281-289, 2002.

[14] H. Kanma, N. Wakabayashi, R. Kanazawa, and H. Ito, "Home appliance control system over Bluetooth with a cellular phone," IEEE Trans. Consum. Electron., vol. 49, no. 4, pp. 1049-1053, 2003. 
International Journal of Computer Applications (0975 - 8887)

Volume 179 - No.16, January 2018

[15] G. Barbon, M. Margolis, F. Palumbo, F. Raimondi, and N. Weldin, "Taking Arduino to the Internet of Things: The ASIP programming model," Elsiver, Comput. Electr. Eng. Commun., vol. 89-90, pp. 128-140, 2016.

[16] R. Pi, DATASHEET Raspberry Pi Compute Module (CM1) Raspberry Pi Compute Module 3 (CM3)
Raspberry Pi Compute Module 3 Lite (CM3L), vol. 3, no. October. 2016.

[17] A Wiley Brand, Raspberry Pi FOR DUMMIES. 2013.

[18] ESP8266 Datasheet, "ESP8266EX Datasheet," Espr. Syst. Datasheet, pp. 1-31, 2015. 